\title{
Brain Shift Correction Based on a Boundary Element Biomechanical Model with Different Material Properties
}

\author{
Olivier Ecabert ${ }^{1,2}$, Torsten Butz ${ }^{3}$, Arya Nabavi ${ }^{4}$, and Jean-Philippe Thiran ${ }^{3}$ \\ 1 Darmstadt University of Technology, 64283 Darmstadt, Germany \\ 2 Philips Research, Weisshausstrasse 2, 52066 Aachen, Germany \\ olivier.ecabert@philips.com \\ 3 Swiss Federal Institute of Technology (EPFL), Signal Processing Institute \\ 1015 Lausanne, Switzerland \\ torsten.butz, JP.Thiran@epfl.ch \\ 4 University of Kiel, Department of Neurosurgery, 24106 Kiel, Germany \\ arya@bwh.harvard.edu
}

\begin{abstract}
Neuronavigation systems are usually subject to inaccuracy due to intraoperative changes like brain shift or tumor resection. In order to correct for these deformations a biomechanical model of the brain is proposed. Not only elastic tissues, but also fluids are modeled, since an important volume of the head contains cerebrospinal fluid, which does not behave like soft tissues. Unlike other approaches, we propose to solve the differential equations of the model by means of the boundary element method, which has the advantage of only considering the boundaries of the different biomechanically homogeneous regions. The size of the matrix to invert is therefore drastically reduced. Finally, our method is assessed with sequences of intraoperative MR images, showing better performances for the elastic/fluid model than for the purely elastic one.
\end{abstract}

\section{Introduction}

Neuronavigation systems are used intraoperatively to help the surgeon guide and ascertain the position of his tools within the patient's head. Over the last decade the development of low-cost high-performance computers, along with a constant improvement of imaging modalities (e.g. MRI, CT), have enabled the routine use of frameless image-guided stereotactic systems in the operating room. Their application fields are wide, including for example tumor resection, endoscopy and radiosurgery.

Although these systems allow a precise initial navigation (within $1 \mathrm{~mm}$ ), they are usually subject to a progressive degradation in accuracy during the operation $[1,2,3,4]$. Indeed, both frame-based and frameless systems assume that the preoperatively acquired images and the physical space can be registered by a rigid transformation. This is only valid for the first steps of the operation but after some surgical manipulations like dura opening, cerebrospinal fluid (CSF) drainage or tumor resection $[1,4]$, the amount of brain shift is likely to increase. 
Brain shifts reaching more than $7 \mathrm{~mm}$ and sometimes up to $20 \mathrm{~mm}$ have been reported in $[2,4,5]$.

The largest inaccuracy of the stereotactic systems is reached near the end of the surgery. It is also the most critical stage, since the deep structures are resected, diminishing the efficiency of the currently used neuronavigation systems. It is therefore of great importance to intraoperatively correct for these deformations. To address this problem three main approaches have been developed. The first proposed method consists of updating the whole image data with intraoperative CT or MR scanners. It has the advantage that the image space always reflects the actual anatomy, but these devices remain expensive, are usually dedicated for operative use only, and interfere with access to the operative field $[2,6]$.

The second approach relies on the simulation of the intraoperative brain behavior based on a computational model of brain tissue deformation [6,7]. Paulsen et al. [6] modeled the brain as an elastic body with an interstitial fluid, while solving the resulting differential equations with the finite element method (FEM). Another work was reported in [7], which considers the brain as a damped springmass model. Although both models models simulate the brain deformation under the action of the gravity and after opening the dura, they are not able to deal with the deformation resulting from surgical manipulations. Moreover, boundary conditions (surface forces, skull/brain interactions) are difficult to model. More recently, Miga et al. [8] extended the model [6] to account for retraction and resection during surgery within the FEM framework.

The third approach is a kind of compromise between the both mentioned above. Indeed, some information is intraoperatively measured and applied like an initial condition to a biomechanical tissue model, in order to fit the preoperative images to the actual anatomy. In this context, Ferrant et al. [9] proposed to register intraoperative MR images by matching object surfaces (ventricles, cortical surface) and then computing the internal deformation using an elastic model. A similar two-steps approach is proposed in [10] where the biomechanical model is guided by a pair of stereo cameras, which intraoperatively measure the exposed brain surface. In both approaches, the biomechanical equations are solved with the FEM. By combining intraoperative measurements with preoperatively acquired high quality images, it is possible to improve the accuracy of current navigation systems with low-cost equipments.

The method proposed in the present paper belongs to the third category and most of the efforts were focused on the tissue modeling and on the computing method to solve the biomechanical equations:

- Unlike usual approaches, we choose to solve the biomechanical equations by means of the boundary element method (BEM) [11] instead of the FEM. The BEM has the advantage of only considering the boundaries of the different regions, reducing therefore the problem dimension by one, and thus drastically the size of the matrix to invert. Nevertheless, the computed biomechanical behavior remains exactly the same as that of the original volumetric system. 
- Inspired from [12] and in order to increase the accuracy of the correction, different physical models are considered for the brain tissues and cerebrospinal fluid, which are respectively simulated with the Navier and Stokes equations.

Finally, experiments are carried out on sequences of intraoperative MR (IMR) images, in order to assess and validate the proposed method. This imaging modality is ideal for that task, since it provides reasonable spatial and contrast resolution.

\section{$2 \quad$ Tissue Modeling}

The biomechanical tissue modeling is based on the theory of continuum mechanics [13]. This is a general framework, which describes the equilibrium and the relationship between forces and displacements within a material, irrespective to its physical nature (e.g. elastic). The specific material properties are specified through the constitutive equations. They are the link between the stress and strain inside the body, and the general relationships mentioned above.

Two biomechanical models are considered in our work, namely linear elastic materials for the brain tissues, and Stokes flows for the CSF. We refer to [12] for a complete description. The biomechanical tissue relationships are the Navier equations for the linear elasticity

$$
G u_{i, j j}+\frac{G}{1-2 \nu} u_{j, j i}+b_{i}=0,
$$

and the Stokes equations for the incompressible fluid model

$$
\begin{aligned}
& \mu^{*} u_{i, j j}-p_{, i}+b_{i}=0 \\
& u_{j, j}=0,
\end{aligned}
$$

where the index notation was used and $u_{i}, p_{i}, b_{i}(i=1,2)$ are the components of the displacement, traction and body force (e.g. gravity) vectors respectively. $G$ stands for the shear modulus and $\nu$ for the Poisson ratio. $\mu^{*}$ is the dynamic viscosity and is related to the standard fluid viscosity $\mu$ by $\mu^{*}=\mu / \delta t$ where $\delta t$ is the deformation time interval $[12,13]$.

\section{Approximate Method}

Unlike $[6,9,10,12]$, where the differential equations are discretized and solved with the finite element method (FEM), our approach relies on the boundary element integral formulation [11]. It has the advantage of only necessitating the discretization of the domain boundaries, reducing therefore strongly the size of the matrix to invert (see Figure 1). Unfortunately, the resulting matrix does not remain diagonal banded any more as for the FEM. This property was extensively used in [9] to design a fast linear system solving algorithm. The matrix size ratio between the BEM and FEM depends on the geometry under consideration. However, when coupling different homogenous regions with the BEM, the matrix becomes partially sparse and the required storage amount can be reduced. 


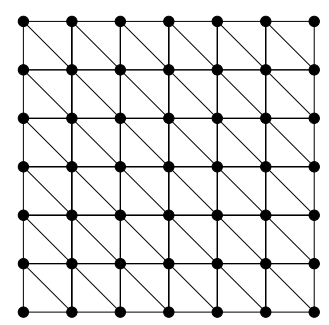

FEM

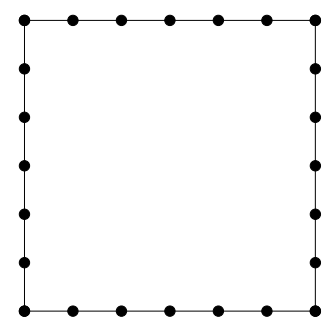

BEM

Fig. 1. 2D domain discretization for the finite and boundary element methods.

\subsection{Navier Equation}

The boundary integral formulation for the elastic model (cf. Equation (1)) is given by [11]

$$
\begin{aligned}
& c_{i j}(\mathbf{x}) u_{j}(\mathbf{x})= \\
& \int_{\partial D} u_{i j}^{*}(\mathbf{x}, \mathbf{y}) p_{j}(\mathbf{y}) d \Gamma_{\mathbf{y}}-\int_{\partial D} p_{i j}^{*}(\mathbf{x}, \mathbf{y}) u_{j}(\mathbf{y}) d \Gamma_{\mathbf{y}}+\int_{D} u_{i j}^{*}(\mathbf{x}, \mathbf{y}) b_{j}(\mathbf{y}) d \Omega_{\mathbf{y}}
\end{aligned}
$$

where $c_{i j}(\mathbf{x})$ is a coefficient, whose value depends on the position $\mathbf{x} . D$ and $\partial D$ refer respectively to the integration domain and its boundary. $u_{i j}^{*}(\mathbf{x}, \mathbf{y})$ and $p_{i j}^{*}(\mathbf{x}, \mathbf{y})$ are the fundamental solutions of Equation (1) and take the following forms for the two-dimensional (2D) case:

$$
\begin{gathered}
u_{i j}^{*}(\mathbf{x}, \mathbf{y})=-\frac{1}{8 \pi(1-\nu) G}\left\{(3-4 \nu) \ln (r) \delta_{i j}-r_{, i} r_{, j}\right\} \\
p_{i j}^{*}(\mathbf{x}, \mathbf{y})=-\frac{1}{4 \pi(1-\nu) r}\left\{\left[(1-2 \nu) \delta_{i j}+2 r_{, i} r_{, j}\right] \frac{\partial r}{\partial n}-(1-2 \nu)\left(r_{, i} n_{j}-r_{, j} n_{i}\right)\right\}
\end{gathered}
$$

with $r=\sqrt{\left(x_{1}-y_{1}\right)^{2}+\left(x_{2}-y_{2}\right)^{2}}$ and $n_{i}$ the normal vector to the boundary. The last surface integral of Equation (3) can be transformed into a curve integral by means of the Galerkin tensor [11]. This yields

$$
\int_{D} u_{i j}^{*}(\mathbf{x}, \mathbf{y}) b_{j}(\mathbf{y}) d \Omega_{\mathbf{y}}=\int_{\partial D} \frac{-r}{8 \pi G}\left\{(2 \ln (r)+1)\left(b_{i} n_{k} r_{, k}-\frac{1}{2(1-\nu)} b_{k} r_{, k} n_{i}\right)\right\} d \Gamma_{\mathbf{y}} .
$$

\subsection{Stokes Equation}

The details for the boundary integral formulation of the Stokes flow may be found in [14]. The boundary integral referring to Equation (2) is

$$
\begin{aligned}
& c_{i j}(\mathbf{x}) u_{j}(\mathbf{x})= \\
& \int_{\partial D} u_{i j}^{*}(\mathbf{x}, \mathbf{y}) p_{j}(\mathbf{y}) d \Gamma_{\mathbf{y}}-\int_{\partial D} K_{i j}^{*}(\mathbf{x}, \mathbf{y}) u_{j}(\mathbf{y}) d \Gamma_{\mathbf{y}}+\int_{D} u_{i j}^{*}(\mathbf{x}, \mathbf{y}) b_{j}(\mathbf{y}) d \Omega_{\mathbf{y}}
\end{aligned}
$$


with the fundamental solutions for the $2 \mathrm{D}$ case

$$
\begin{gathered}
u^{*}(\mathbf{x}, \mathbf{y})=-\frac{1}{4 \pi \mu^{*}}\left\{\ln (r) \delta_{i j}-r_{, i} r_{, j}\right\} \\
K_{i j}^{*}(\mathbf{x}, \mathbf{y})=\frac{1}{\pi r} r_{, i} r_{, j} r_{, k} n_{k} .
\end{gathered}
$$

The same remark as for the elastic case applies for the surface integral of Equation (4), leading to

$$
\int_{D} u_{i j}^{*}(\mathbf{x}, \mathbf{y}) b_{j}(\mathbf{y}) d \Omega_{\mathbf{y}}=\int_{\partial D} \frac{-r}{8 \pi \mu^{*}}\left\{(2 \ln (r)+1)\left(b_{i} n_{k} r_{, k}-b_{k} r_{, k} n_{i}\right)\right\} d \Gamma_{\mathbf{y}} .
$$

All the surface integrals are now transformed into curve integrals, which can be discretized using linear isoparametric elements since they proved to give satisfactory accuracy without requiring too much computing efforts [11]. After coupling the different homogenous regions (brain tissues and ventricles) with the compatibility and equilibrium conditions [12], the resulting system becomes a standard system of linear equations. Solving this system returns the missing boundary displacements or tractions. All unknown displacements are now determined on the boundary, and the internal displacements can be computed in a second step at any arbitrary position x, using Equations (3) and (4) respectively.

\section{Results and Discussion}

In this section, the accuracy of the proposed elastic/fluid biomechanical model is assessed on 2D slices of 3D intraoperative MR images $(0.5$ Tesla, $256 \times 256 \times 60$, $0.9375 .9375 \times 2.5 \mathrm{~mm}^{3}$ ), and compared to a purely elastic model. Experiments are carried out on two data sets of two different patients. The columns (a) of Figures 2 and 3 depict the actual brain anatomy at two time points, reflecting the deformation occurring during the surgery. The small white dots superimposed on the images represent the initial cortical and ventricle boundaries prior to the dura opening. An important shift is visible at the top of the brain for both cases. Moreover, a clear loss of CSF can be observed.

The experiments were carried out as follows: First, the images are rigidly registred by maximizing their mutual information [15] to remove eventual intraoperative patient's movements. Second, the brain boundaries are extracted using a geodesic active contour model [16]. The appropriate constitutive equation is then given to the corresponding region defined by the extracted boundaries. The model parameters are $G=112^{\prime} 000 \mathrm{~N} / \mathrm{m}^{2}$ and $\nu=0.4$ for the elastic material, and $\mu^{*}=0.01 \mathrm{~N} / \mathrm{m}^{2}$ for the Stokes flow. They reflect the mean values of brain and skull elastic coefficients from a comprehensive review published in [12]. The cortical (outter) boundaries are matched, looking for the smallest distance between the initial boundary to the actual one. Finally, the resulting boundary displacements are used as input (initial conditions) to the biomechanical model 

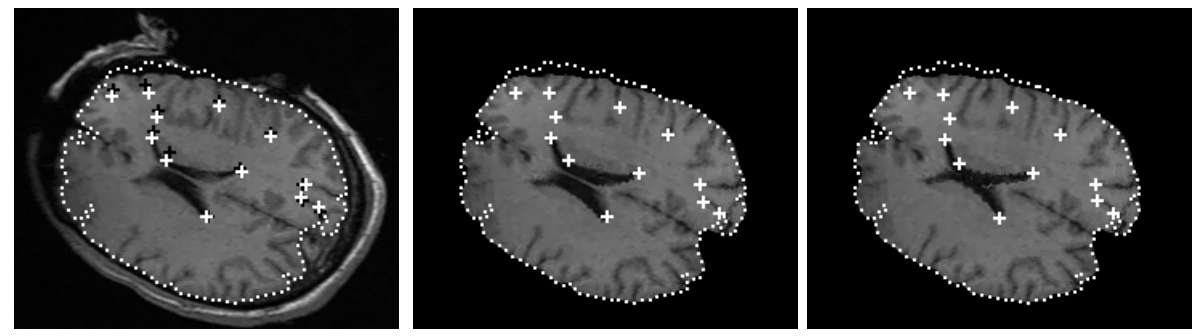

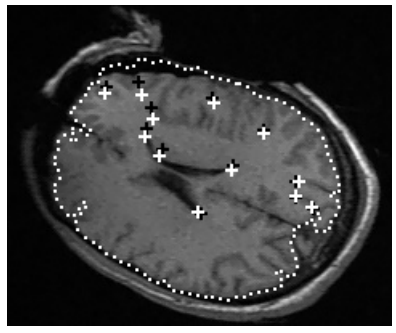

(a)

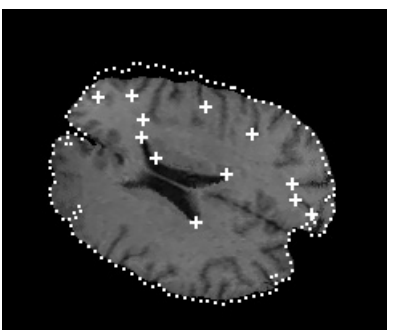

(b)

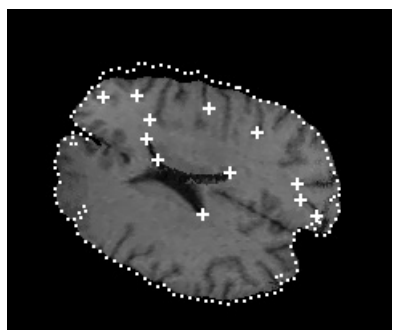

(c)

Fig. 2. Case 1. (a) Brain deformation at two time points during surgery. (Black crosses: Initial landmarks, white crosses: Actual landmarks.) Correction using the purely elastic (b) and the elastic/fluid model (c). (White crosses: Landmarks after deformation.)
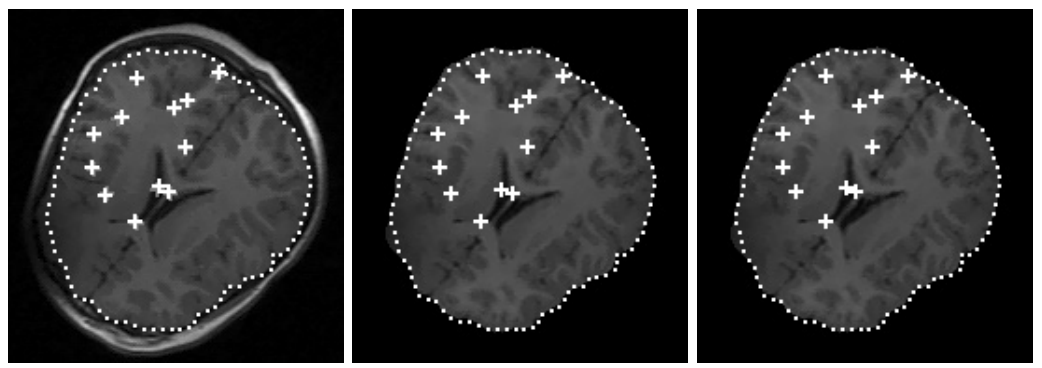

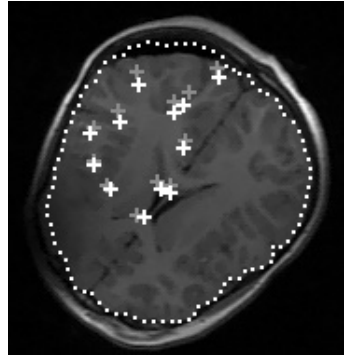

(a)

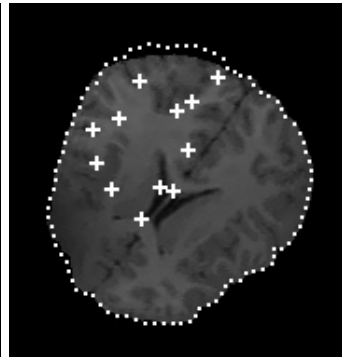

(b)

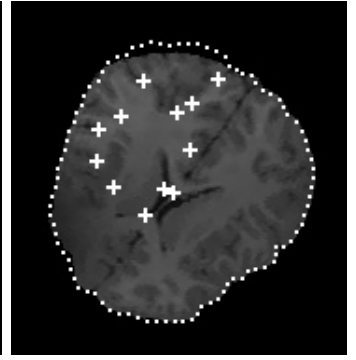

(c)

Fig. 3. Case 2. (a) Brain deformation at two time points during surgery. (Gray crosses: Initial landmarks, white crosses: Actual landmarks.) Correction using the purely elastic (b) and the elastic/fluid model (c). (White crosses: Landmarks after deformation.) 
and after solving the linear system, the internal displacements can be computed for the whole brain.

Brain shift correction results can be seen on Figures 2 and 3, column (b) for the purely elastic model, and column (c) for the model simulating the CSF as an incompressible viscous flow. As quantitative measure, we propose to use the mean value of the distances (errors) computed between 12 landmarks identified on the preoperative and actual scans. Note that none of the landmarks was situated on the cortical boundary, since it would be perfectly registered during the boundary matching, and the resulting error would be unrealistically small. The mean errors are reported in the tables below for the different cases.

\begin{tabular}{|l||c|c|c|c|}
\hline Case 1 & Max. Shift & Mean Error & Elastic & Elastic + Fluid \\
\hline \hline 1st Time Point & $4.5 \mathrm{~mm}$ & $3.0 \mathrm{~mm}$ & $1.8 \mathrm{~mm}$ & $1.8 \mathrm{~mm}$ \\
\hline 2nd Time Point & $5.1 \mathrm{~mm}$ & $3.4 \mathrm{~mm}$ & $2.4 \mathrm{~mm}$ & $2.3 \mathrm{~mm}$ \\
\hline
\end{tabular}

\begin{tabular}{|l||c|c|c|c|}
\hline Case 2 & Max. Shift & Mean Error & Elastic & Elastic + Fluid \\
\hline 1st Time Point & $1.8 \mathrm{~mm}$ & $1.4 \mathrm{~mm}$ & $1.4 \mathrm{~mm}$ & $1.3 \mathrm{~mm}$ \\
\hline 2nd Time Point & $7.5 \mathrm{~mm}$ & $4.5 \mathrm{~mm}$ & $2.3 \mathrm{~mm}$ & $1.6 \mathrm{~mm}$ \\
\hline
\end{tabular}

The first column indicates the maximum shift between the initial and actual (i.e. not deformed) images. The mean error is reported in the second column when no correction is performed. The third and fourth columns show the mean error for the purely elastic and inhomogeneous elastic/fluid models respectively.

A maximum error up to $7.5 \mathrm{~mm}$ was observed and compensated on the cortical boundary. In all cases the mean error could be decreased by the use of a biomechanical model, with the smaller residual error for the elastic/fluid model (mean correction up to $2.9 \mathrm{~mm}$ ). Note also the better behavior near the ventricles. These results act therefore favorably for using an inhomogeneous model and for simulating the CSF with the appropriate equation.

The programs were written in $\mathrm{C}++$ and the simulations were run on a Pentium III, 1.1 GHz. The boundaries were discretized with about 500 points for the cortical boundary and 100 points for each ventricle, leading to matrices of size $1400 \times 1400$ (two coordinates per point). The linear system was solved in about 0.3 second and the internal displacements were computed in about 1 second, corresponding to a reasonable computation time for such an application.

\section{Concluding Remarks}

This paper introduces a non-homogeneous biomechanical model of the brain combining elastic materials and fluids to compensate for the progressive shift occurring during surgery. Our model is driven by correspondences on the cortical surface, and through experiments carried out on real medical data, the elastic/fluid model shows to perform better than a purely elastic one.

In order to reduce the computation time, the boundary element method (BEM) is used to solve the system of differential equations. The BEM only considers the boundaries of homogeneous regions, reducing therefore the problem 
dimension by one, and thus drastically the size of the matrix to invert. Nevertheless, the computed biomechanical behavior remains exactly the same as for the original volumetric system. Although BEM does have strong advantages, anisotropy and especially white/gray matter inhomogeneity may be complex to address effectively with BEM. Finally, brain shift is a complex 3D phenomenon and, although our 2D approach shows to be effective in correcting for intraoperative brain changes, a 3D model should even capture the deformation better. $3 \mathrm{D}$ modeling is the future direction of our investigation.

\section{References}

1. Nabavi, A., Black, P.M., Gering, D.T., Westin, C.F., Metha, V., Pergolizzi, R.S., Ferrant, M., Warfield, S.K., Hata, N., Schwartz, R., Wells, W., Kikinis, R., Jolesz, F.A.: Serial intraoperative MR imaging of brain shift. Neurosurgery 48 (2001)

2. Bucholz, R.D., Yeh, D.D., Trobaugh, J., McDurmont, L.L., Sturm, C.D., Baumann, C., Henderson, J.M., Levy, A., Kessman, P.: The correction of stereotactic inaccuracy caused by brain shift using an intraoperative ultrasound device. In: CVRMed-MRCAS '97, Springer (1997) 459-466

3. Maurer, C.R., Hill, D.L.G., Martin, A.J., Liu, H., McCue, M., Rueckert, D., Lloret, D., Hall, W.A., Maxwell, R.E., Hawkes, D.J., Truwit, C.L.: Investigation of intraoperative brain deformation using a 1.5-T interventional MR system: Preliminary results. IEEE Transactions on Medical Imaging 17 (1998) 817-825

4. Nimsky, C., Ganslandt, O., Cerny, S., Hastreiter, P., Greiner, G., Fahlbusch, R.: Quantification of, visualization of, and compensation for brain shift using intraoperative magnetic resonance imaging. Neurosurgery 47 (2000) 1070-1080

5. Hill, D.L., Maurer, C.R., Wang, M.Y., Maciunas, R.J., Barwise, J.A., Fitzpatrick, J.M.: Estimation of intraoperative brain surface movement. In Troccaz, J., Grimson, E., Mosges, R., eds.: CVRMed-MRCAS '97. Volume 1205 of LNCS., (Springer)

6. Paulsen, K.D., Miga, M.I., Kennedy, F.E., Hoopes, P.J., Hartov, A., Roberts, D.W.: A computational model for tracking subsurface tissue deformation during stereotactic neurosurgery. IEEE Transactions on Biomedical Engineering 46 (1999)

7. Škrinjar, O.M., Duncan, J.S.: Real time 3d brain shift compensation. In: MICCAI '99. Volume 1679 of Lecture Notes in Computer Science., Springer (1999)

8. Miga, M. et al.: Modeling of retraction and resection for intraoperative unpdating of images during surgery. Neurosurgery 49 (2001)

9. Ferrant, M., Nabavi, A., Macq, B., Jolesz, F.A., Kikinis, R., Warfield, S.K.: Registration of 3-D intraoperative MR images of the brain using a finite-element biomechanical model. IEEE Transaction on Medical Imaging 20 (2001) 1384-1397

10. Škrinjar, O.M., Studholme, C., Nabavi, A., Duncan, J.S.: Steps toward a stereocamera-guided biomechanical model for brain shift compensation. In: IPMI 2001. Volume 2082 of Lecture Notes in Computer Science., Springer (2001) 183-189

11. Brebbia, C.A., Telles, J.C.F., Wrobel, L.C.: Boundary Element Techniques. Theory and Applications in Engineering. Springer, Berlin (1984)

12. Hagemann, A., Rohr, K., Stiehl, H.: Coupling of fluid and elastic models for biomechanical simulations of brain deformations using FEM. Medical Image Analysis 6 (2002) 375-388

13. Fung, Y.: A First Course in Continuum Mechanics. Prentice-Hall, Engelwood Cliffs, N.J. (1969) 
14. Power, H., Wrobel, L.: Boundary Integral Methods in Fluid Mechanics. Computational Mechanics Computation, Southampton, U.K. (1995)

15. Maes, F., Colligno, A., Vandermeulen, D., Marchal, G., Sueten, P.: Multimodality image registration by maximization of mutual information. IEEE Transactions on Medical Imaging 16 (1997) 187-198

16. Ecabert, O., Thiran, J.P.: Variational image segmentation by unifying region and boundary information. In: ICPR, Quebec City, Canada (2002) 885-888 